\title{
Quantum Dynamics in the Fermi-Pasta-Ulam Problem
}

\author{
Gennady Berman \\ Los Alamos National Laboratory \\ MS B213 \\ Los Alamos \\ NM 87545 \\ U.S.A.
}

\author{
Nikolai Tarkhanov \\ University of Potsdam \\ Institute of Mathematics \\ P.O. Box 601553 \\ 14415 Potsdam, \\ Germany
}

March 16, 2004

\begin{abstract}
We study the dynamics of four wave interactions in a nonlinear quantum chain of oscillators under the "narrow packet" approximation. We determine the set of times for which the evolution of decay processes is essentially specified by quantum effects. Moreover, we highlight the quantum increment of instability.
\end{abstract}

\section{Introduction}

In a nonlinear environment with dispersion waves may be instable under decay processes, cf. for instance [11]. The instability is observed by effective interaction of waves with vectors $\vec{k}_{j}$ and frequencies $\omega\left(\vec{k}_{j}\right)$ in a neighbourhood of resonances

$$
\begin{aligned}
\sum_{j} n_{j} \omega\left(\vec{k}_{j}\right) & =0, \\
\sum_{j} n_{j} \vec{k}_{j} & =0,
\end{aligned}
$$

whence the wave amplitudes change exponentially fast in time at the initial stage, and nonlinear effects turn out to be essential for describing their dynamics.

The wave decay processes are of considerable interest in problems of chemistry, hydrodynamics of liquid and gas, plasma physics, nonlinear optics, solid physics, etc. 
Usually the dynamics of wave decay processes is described in the framework of classical approach. Such approach seems to be justified so far the energy of interacting waves is sufficiently large and effects related to quantities of order $\hbar$ are not in time for becoming transparent. However, it is not always possible to neglect the influence of quantum mechanics corrections on system dynamics even in the quasi-classical setting, if particularly the classical approximation is instable. The study of dynamical stochasticity in classical and quantum mechanics shows that if the classical system is strongly instable then its quantum dynamics may essentially differ from the classical one, cf. [3], [5], [12].

The present paper is devoted to quantum mechanics analysis of the dynamics of decay processes of type (0.1) which occur in a one-dimensional nonlinear chain of connected oscillators, cf. [8]. The Hamiltonian of the system has the form

$$
H=\sum_{n=1}^{N}\left(\frac{p_{n}^{2}}{2 m}+\frac{\epsilon}{2}\left(u_{n+1}-u_{n}\right)^{2}+\frac{\nu}{4}\left(u_{n+1}-u_{n}\right)^{4}\right),
$$

where $p_{n}$ is the momentum of the $n$th oscillator, $u_{n}$ the displacement of the $n$th oscillator from the equilibrium position, $N$ is the number of oscillators, $\epsilon$ is an elasticity constant, $\nu$ is the parameter of nonlinearity, and $m$ is the mass of an oscillator. In the sequel the boundary conditions are chosen to be periodic, i.e., $p_{n+N}=p_{n}$ and $u_{n+N}=u_{n}$.

The system $(0.2)$ for $\hbar=0$ is one of the simplest models for finding conditions of appearance of stochastic properties in nonlinear systems with many degrees of freedom. It is intensively investigated beginning with 1955, cf. [8], [6], etc. There is certain connection between the instability of the decay type in question and the stochastic instability of [4]. This latter paper presents a numerical investigation of system $(0.1)$ in the case of initiating short waves ("narrow packet" approximation). If the parameter $\nu$ exceeds a critical value $\nu_{c}$ four wave decay processes seem to appear corresponding to resonances $(0.1)$. Under further increase of $\nu$ diverse resonances of type (0.1) might interact with each other, which finally results in a stochastic behaviour of the chain. The availability of decay processes in a classical chain seems thus to be a preliminary step to arising a stochastic instability in the system.

Hence the study of the dynamics of four wave decay processes for system (0.2) in the quantum case seems to be well motivated. This paper is organised as follows. In Section 1 we have compiled some basic facts on the dynamics of four wave decay processes in a classical chain. The equations describing the dynamics of quantum decay are presented in Section 2. Sections 3, 4 and 5 contain a detailed study of the quantum decay system. The local solution of the system guaranteed by the Cauchy-Kovalevskaya Theorem is proved to analytically extend to all time, space and parameter values. In Section 6 we treat the asymptotic behaviour of the solution for large times. It is shown in particular that quantum effects lead to slowing down the rate of the develop- 
ment of decay instability as compared with the classical case. Moreover, the quantum effects force the disappearance of the critical value $\nu_{c}$.

\section{Classical limit}

Before discussing the decay instability in the quantum case we look more closely at some peculiarities of the dynamics of four wave decay processes in a classical chain. To this end, we pass in (0.2) to the canonical variables $a_{k}$ and $a_{k}^{*}$ by

$$
a_{k}=\frac{1}{\sqrt{2 m \hbar \omega_{k}}}\left(P_{k}-\imath \omega_{k} U_{k}^{*}\right)
$$

where

$$
\begin{aligned}
P_{k} & =\frac{1}{\sqrt{N}} \sum_{n=1}^{N} p_{n} e^{-2 \pi \frac{k}{N} n \imath}, \\
U_{k} & =\frac{1}{\sqrt{N}} \sum_{n=1}^{N} u_{n} e^{2 \pi \frac{k}{N} n \imath}, \\
\omega_{k} & =2 \sqrt{\frac{\epsilon}{m}} \sin \pi \frac{k}{N} .
\end{aligned}
$$

In the classical case, the commutator $\left[a_{j}, a_{k}^{\dagger}\right]=0$ vanishes, and $I_{k}=\hbar\left|a_{k}\right|^{2}$ is a classical action of the phonon with momentum $k$.

Suppose that the initial data of system (0.2) satisfy the condition of "narrow packet" approximation

$$
\delta k / k_{0} \ll 1,
$$

where $\delta k=\left|k-k_{0}\right|$ is the characteristic size of a packet of initiated modes by $k$, and $k_{0}$ is the characteristic wave number of the packet $\left(k_{0} \sim N / 2\right.$, the number of oscillations of anti-phase oscillations). In the variables $a_{k}, a_{k}^{*}$ the Hamiltonian (0.2) takes the form

$$
\begin{aligned}
H & =\hbar \sum_{k=1}^{N} \omega_{k} a_{k}^{*} a_{k}+\frac{1}{2} \hbar^{2} \sum_{k_{1}, k_{2}, k_{3}, k_{4}} V_{k_{1} k_{2} k_{3} k_{4}} a_{k_{1}}^{*} a_{k_{2}}^{*} a_{k_{3}} a_{k_{4}} \delta_{k_{1}+k_{2}-k_{3}-k_{4}, 0} \\
& +O(1)
\end{aligned}
$$

where

$$
V_{k_{1} k_{2} k_{3} k_{4}}=\frac{3 \nu}{\epsilon m N}\left(\sin \pi \frac{k_{1}}{N} \sin \pi \frac{k_{2}}{N} \sin \pi \frac{k_{3}}{N} \sin \pi \frac{k_{4}}{N}\right)^{1 / 2} .
$$

In (1.3) the terms $a_{k_{1}}^{*} a_{k_{2}}^{*} a_{k_{3}} a_{k_{4}}$ represent the resonance four wave interaction processes of modes, which are decisive under the condition (1.2). By $O(1)$ are meant the non-resonance terms like $a_{k_{1}} a_{k_{2}} a_{k_{3}} a_{k_{4}}, a_{k_{1}}^{*} a_{k_{2}} a_{k_{3}} a_{k_{4}}$, etc., which can 
be neglected under the approximation in question, at least at the initial stage. Under the condition (1.2) one can set

$$
\begin{aligned}
\omega_{k} & \approx \omega_{k_{0}}+c\left(k-k_{0}\right)-\Omega\left(k-k_{0}\right)^{2}, \\
V_{k_{1} k_{2} k_{3} k_{4}} & \approx V_{0}
\end{aligned}
$$

where

$$
c=2 \sqrt{\frac{\epsilon}{m}} \frac{\pi}{N} \cos \pi \frac{k_{0}}{N}, \quad \Omega=\sqrt{\frac{\epsilon}{m}}\left(\frac{\pi}{N}\right)^{2} \sin \pi \frac{k_{0}}{N}, \quad V_{0}=\frac{3 \nu}{\epsilon m N}\left(\sin \pi \frac{k_{0}}{N}\right)^{2} .
$$

Substituting (1.3) and (1.4) into the equations of motion

$$
\imath \dot{a}_{k}=\frac{\partial H}{\partial a_{k}^{*}}
$$

we get

$$
\imath \dot{A}_{j}=-j^{2} \Omega A_{j}+\hbar V_{0} \sum_{j_{2}, j_{3}, j_{4}} A_{j_{2}}^{*} A_{j_{3}} A_{j_{4}} \delta_{j+j_{2}-j_{3}-j_{4}, 0},
$$

where

$$
A_{j}=\exp \left(\left(\omega_{k_{0}}+c j\right) t \imath\right) a_{j+k_{0}} .
$$

The equation (1.5) describes the dynamics of four wave interactions in chain (0.2). As is shown in [4], if $\nu \ll 2 \pi^{2} k_{0} / 3 N E_{k_{0}} \sim 1 / E, E$ being the energy of the system, then the "narrow packet" approximation survives in the course of time. It follows that equations (1.5) actually simulate the dynamics of (0.2) for all times. In the sequel we think of equations (1.5) as the input ones.

We next present a condition for arising the decays. It is easy to verify that the equation (1.5) has an explicit solution of the form of finite amplitude wave

$$
\begin{aligned}
& A_{k}(t)=\exp \left(\left(\Omega_{k}-\hbar V_{0}\left|A_{k}\right|^{2}\right) t \imath\right) A_{k}, \\
& A_{j}(t)=0, \quad \text { if } j \neq k,
\end{aligned}
$$

where $\Omega_{k}=k^{2} \Omega$. Let us examine the stability of solution (1.6) with respect to the decay in neighboring modes $2 k \mapsto(k-l)+(k+l)$. Suppose that the modes with $j \neq k$ are slightly perturbed at the initial instant, so that $\left|A_{j}\right| \ll\left|A_{k}\right|$. By linearising equations (1.5) in $A_{j}$ one easily arrives at the system

$$
\begin{aligned}
\dot{A}_{k} & \left.=-\Omega_{k} A_{k}+\hbar V_{0}\left|A_{k}\right|^{2}\right) A_{k}, \\
\imath \dot{A}_{k-l} & =-\Omega_{k-l} A_{k-l}+2 \hbar V_{0}\left|A_{k}\right|^{2} A_{k-l}+\hbar V_{0} A_{k}^{2} A_{k+l}^{*}, \\
\dot{A}_{k+l} & =-\Omega_{k+l} A_{k+l}+2 \hbar V_{0}\left|A_{k}\right|^{2} A_{k+l}+\hbar V_{0} A_{k}^{2} A_{k-l}^{*} .
\end{aligned}
$$

These equalities show that the dynamics of a "large" wave does not change at first approximation of perturbation theory. The amplitudes of "small" waves grow exponentially with the increment

$$
\lambda_{l}=\sqrt{V_{0} I(\Delta \Omega)-\left(\frac{\Delta \Omega}{2}\right)^{2}}=l \Omega \sqrt{\frac{2 V_{0} I}{\Omega}-l^{2}},
$$


where $\Delta \Omega=\Omega_{k-l}+\Omega_{k+l}-2 \Omega_{k}=2 l^{2} \Omega$ is a destruction characteristic of resonance (0.1), and $I=\hbar\left|A_{k}\right|^{2}$. From (1.8) we get the desired condition for existence of decays, namely $2 V_{0} I / \Omega>1$. In terms of original system $(0.2)$ this condition reads

$$
\nu>\frac{\pi^{2}}{3 N E_{k_{0}}} \sim \frac{1}{N E}
$$

\section{Quantum equations of decay}

We now pass to analysis of the quantum case where $p_{n}$ and $u_{n}$ in $(0.2)$ are operators with commutativity relation $\left[u_{j}, p_{k}\right]=\imath \hbar \delta_{j k}$. Changing the variables by (1.1) and (1.4) as in the classical case (see [1] for more details), we get the following system of operator equations which describe the four wave interactions in the quantum case

$$
\imath \dot{A}_{j}=-j^{2}(1+q) \Omega A_{j}+\hbar V_{0} \sum_{j_{2}, j_{3}, j_{4}} A_{j_{2}}^{\dagger} A_{j_{3}} A_{j_{4}} \delta_{j+j_{2}-j_{3}-j_{4}, 0},
$$

where

$$
\begin{aligned}
{\left[A_{j}, A_{k}^{\dagger}\right] } & =\delta_{j k}, \\
q & =\hbar \frac{\nu \cot \frac{\pi}{2 N}}{32 N \sqrt{m \epsilon^{3}}},
\end{aligned}
$$

$\Omega$ and $V_{0}$ being defined in (1.4). The renormalisation of the frequency $\Omega$ is explained by the ordering of operators. It will cause no confusion if we use the same letter $\Omega_{j}$ to designate $j^{2}(1+q) \Omega$.

To treat the system (2.1) we use the techniques of projection onto the basis of coherent states, $\mathrm{cf}$. [2] and [10]. Assume that at the initial instant each mode of the bosonic field rests on a coherent state described by a number $\alpha_{j}$. We denote

$$
\begin{aligned}
\alpha_{j}(t) & =\left\langle\vec{\alpha}\left|A_{j}(t)\right| \vec{\alpha}\right\rangle \\
& =\alpha_{j}\left(t, \vec{\alpha}, \vec{\alpha}^{*}\right)
\end{aligned}
$$

where $|\vec{\alpha}\rangle$ is the vector of states of the phononic field at the initial instant. From (2.1) it follows that the operator $A_{j}(t)$ satisfies the Heisenberg equation

$$
\imath \hbar \dot{A}_{j}=\left[A_{j}(t), H_{\mathrm{eff}}\right],
$$

with the effective Hamiltonian

$$
H_{\mathrm{eff}}=-\hbar \sum_{k} \Omega_{k} A_{k}^{\dagger} A_{k}+\frac{1}{2} \hbar^{2} V_{0} \sum_{k_{1}, k_{2}, k_{3}, k_{4}} A_{k_{1}}^{\dagger} A_{k_{2}}^{\dagger} A_{k_{3}} A_{k_{4}} \delta_{k_{1}+k_{2}-k_{3}-k_{4}, 0} .
$$


Applying the projection thus yields

$$
\begin{aligned}
\imath \dot{\alpha}_{j}(t) & =\hat{T} \alpha_{j}(t), \\
\alpha_{j}(0) & =\alpha_{j},
\end{aligned}
$$

where

$$
\begin{aligned}
\hat{T} & =-\sum_{k} \Omega_{k}\left(\alpha_{k} \frac{\partial}{\partial \alpha_{k}}-C . C .\right) \\
& +\hbar V_{0} \sum_{k_{1}, k_{2}, k_{3}, k_{4}}\left(\alpha_{k_{1}}^{*} \alpha_{k_{2}} \alpha_{k_{3}} \frac{\partial}{\partial \alpha_{k_{4}}}-C . C .\right) \delta_{k_{1}+k_{2}-k_{3}-k_{4}, 0} \\
& +\frac{1}{2} \hbar V_{0} \sum_{k_{1}, k_{2}, k_{3}, k_{4}}\left(\alpha_{k_{1}} \alpha_{k_{2}} \frac{\partial}{\partial \alpha_{k_{3}}} \frac{\partial}{\partial \alpha_{k_{4}}}-C . C .\right) \delta_{k_{1}+k_{2}-k_{3}-k_{4}, 0},
\end{aligned}
$$

the C.C. meaning complex conjugate terms (cf. [1] for more details).

The equation (2.3) is easily checked to possess a solution of the form of finite amplitude periodic wave

$$
\begin{array}{ll}
\alpha_{k}(t)=\exp \left(\Omega_{k} t \imath-\left(1-\exp \left(-\hbar V_{0} t \imath\right)\right)\left|\alpha_{k}\right|^{2}\right) \alpha_{k}, & \\
\alpha_{j}(t)=0, & \text { if } j \neq k .
\end{array}
$$

Note that the solution (2.4) turns into the classical wave (1.6) when $\hbar \rightarrow 0$, $\left|\alpha_{k}\right| \rightarrow \infty$, and $\hbar\left|\alpha_{k}\right|^{2} \rightarrow I$. We now examine the stability of solution (2.4) relatively to the decay in neighboring modes $2 k \mapsto(k-l)+(k+l)$. Assume that at the initial instant the amplitudes of the modes $j \neq k$ are small, i.e., $\left|\alpha_{j}\right| \ll\left|\alpha_{k}\right|$. In this case one can look for a solution $\alpha_{k+l}$ of (2.4) in the form of expansion in $\alpha_{j}$,

$$
\begin{aligned}
\alpha_{k+l}\left(t, \vec{\alpha}, \vec{\alpha}^{*}\right) & =c_{l, 0}\left(t, \alpha_{k}, \alpha_{k}^{*}\right) \\
& +\sum_{j \neq 0}\left(c_{l, j}^{(1,0)}\left(t, \alpha_{k}, \alpha_{k}^{*}\right) \alpha_{k+j}+c_{l, j}^{(0,1)}\left(t, \alpha_{k}, \alpha_{k}^{*}\right) \alpha_{k+j}^{*}\right) \\
& +\ldots,
\end{aligned}
$$

the dots meaning the terms containing the products $\alpha_{k+j_{1}} \alpha_{k+j_{2}}, \alpha_{k+j_{1}}^{*} \alpha_{k+j_{2}}$, $\alpha_{k+j_{1}}^{*} \alpha_{k+j_{2}}^{*}$, etc. From the initial condition $\alpha_{k+l}\left(0, \vec{\alpha}, \vec{\alpha}^{*}\right)=\alpha_{k+l}$ we readily deduce that

$$
\begin{aligned}
c_{0,0}\left(0, \alpha_{k}, \alpha_{k}^{*}\right) & =\alpha_{k}, & c_{l, 0}\left(0, \alpha_{k}, \alpha_{k}^{*}\right) & =0 ; \\
c_{l, j}^{(1,0)}\left(0, \alpha_{k}, \alpha_{k}^{*}\right) & =\delta_{l j}, & c_{l, j}^{(0,1)}\left(0, \alpha_{k}, \alpha_{k}^{*}\right) & =0
\end{aligned}
$$

for $l \neq 0$. In (2.5) $\alpha_{k+j}$ and $\alpha_{k+j}^{*}$ are the initial amplitudes of "small" waves, and $\alpha_{k}$ the initial amplitude of a "large" wave. The coefficients $c_{l, 0}, c_{l, j}^{(1,0)}$ and 
$c_{l, j}^{(0,1)}$, etc. do not explicitly contain smallness related to the amplitudes $\alpha_{k+j}$ with $j \neq 0$.

Below, we will study the dynamics of functions $c_{l, 0}, c_{l, j}^{(1,0)}$ and $c_{l, j}^{(0,1)}$, for they determine the evolution of small perturbations with amplitudes $\alpha_{k+j}$. Substituting (2.5) into (2.3) and gatherings the coefficients of the same powers of $\alpha_{k+j}$, we arrive at a system of equations for the coefficients which is not closed in general, i.e., the equations for $c_{l, 0}, c_{l, j}^{(1,0)}$ and $c_{l, j}^{(0,1)}$ also include higher order coefficients. However, one can show that higher order coefficients describe the influence of small waves on each other and on the large wave. Hence they do not essentially contribute to the dynamics of the system at the initial stage. A quasi-classical asymptotic of the contribution of higher order coefficients is discussed in [1].

On account of the above remark, we cut off the expansion (2.5) upon the linear terms. In this way we get the following closed system of differential equations

$$
\begin{aligned}
\imath \dot{c}_{l, 0} & =\hat{M} c_{l, 0}, \\
\imath \dot{c}_{l, j}^{(1,0)} & =\hat{M} c_{l, j}^{(1,0)}-\left(\Omega_{k+j}-2 \hbar V_{0}\left|\alpha_{k}\right|^{2}\right) c_{l, j}^{(1,0)}+2 \hbar V_{0} \alpha_{k} \frac{\partial}{\partial \alpha_{k}} c_{l, j}^{(1,0)}-\hbar V_{0} \alpha_{k}^{* 2} c_{l,-j}^{(0,1)}, \\
\imath \dot{c}_{l,-j}^{(0,1)} & =\hat{M} c_{l,-j}^{(0,1)}-\left(\Omega_{k-j}-2 \hbar V_{0}\left|\alpha_{k}\right|^{2}\right) c_{l,-j}^{(0,1)}-2 \hbar V_{0} \alpha_{k} \frac{\partial}{\partial \alpha_{k}} c_{l,-j}^{(0,1)}+\hbar V_{0} \alpha_{k}^{2} c_{l, j}^{(1,0)}
\end{aligned}
$$

cf. [1], where

$$
\hat{M}=-\left(\Omega_{k}-\hbar V_{0}\left|\alpha_{k}\right|^{2}\right) \alpha_{k} \frac{\partial}{\partial \alpha_{k}}+\frac{1}{2} \hbar V_{0} \alpha_{k}^{2} \frac{\partial^{2}}{\partial \alpha_{k}^{2}}-C . C .,
$$

the C.C. stand for complex conjugate terms.

The solution of the first equation (2.7) has the form (2.4) and describes the dynamics of a "large" wave at first approximation. The remaining system of two equations can be further simplified. For this purpose we conclude from (2.6) and the linearity of (2.7) that only two relevant summands in (2.5) are different from zero, namely $c_{l, l}^{(1,0)}$ and $c_{l,-l}^{(0,1)}$. Let us now substitute the unknown functions by

$$
\begin{aligned}
& c_{l, l}^{(1,0)}=\exp \left(-\left(\Omega_{k-l}-2 \Omega_{k}\right) t \imath\right) f, \\
& c_{l,-l}^{(0,1)}=\frac{\alpha_{k}}{\alpha_{k}^{*}} \exp \left(-\left(\Omega_{k-l}-2 \Omega_{k}\right) t \imath\right) g .
\end{aligned}
$$

Under this notation the average of the operator $A_{k+l}(t)$ is

$$
\begin{aligned}
\alpha_{k+l}(t) & =\left\langle\vec{\alpha}\left|A_{k+l}(t)\right| \vec{\alpha}\right\rangle \\
& =\exp \left(-\left(\Omega_{k-l}-2 \Omega_{k}\right) t l\right)\left(\alpha_{k+l} f(t)+\frac{\alpha_{k}}{\alpha_{k}^{*}} \alpha_{k-l}^{*} g(t)\right) .
\end{aligned}
$$


Substituting (2.8) into (2.7) we deduce that both $f$ and $g$ depend only on $\left|\alpha_{k}\right|^{2}$ and satisfy the system

$$
\begin{aligned}
\imath \dot{f} & =\left(2 V_{0} I-\left(\Omega_{k-l}+\Omega_{k+l}-2 \Omega_{k}\right)\right) f+2 \hbar V_{0} I \frac{\partial f}{\partial I}-V_{0} I g, \\
\imath \dot{g} & =V_{0} I f+\hbar V_{0} g
\end{aligned}
$$

with initial data

$$
\begin{aligned}
& f(0)=1, \\
& g(0)=0,
\end{aligned}
$$

where $I=\hbar\left|\alpha_{k}\right|^{2}$ stands for the classical action of the $k$ th mode.

Equations (2.9) describe the decay instability in the quantum case. From now on they will be referred to as equations of quantum decay. In the classical case $\hbar=0$ they can be solved explicitly, which shows once again the exponential growth of "small" waves when the increment $\lambda_{l}$ (1.8) increases, provided that $2 V_{0} I>\Omega$, cf. $\S 1$.

\section{Analysis of quantum equations}

Before we pass to the analysis of equations (2.9) we make necessary simplifications. We reset

$$
\begin{aligned}
f & \mapsto \exp \left(-\hbar V_{0} t \imath\right) f \\
g & \mapsto \exp \left(-\hbar V_{0} t \imath\right) g
\end{aligned}
$$

and $\Omega \mapsto \Omega-V_{0} t$. Moreover, we introduce dimensionless time $t \mapsto t / \Omega$ and dimensionless variable $x=V_{0} I / \Omega$. For simplicity we restrict our attention to the case $l=1$. Then (2.9) takes the form

$$
\begin{aligned}
\imath \dot{f} & =2(x-1) f+2 \varepsilon x \frac{\partial f}{\partial x}-x g \\
\imath \dot{g} & =x f
\end{aligned}
$$

where

$$
\varepsilon=\hbar \frac{V_{0}}{\Omega}
$$

is a quantum parameter.

The system (3.1) is of mixed type with hyperbolic degeneracy on the line $x=0$. The general theory yields merely that (3.1) has a real analytic solution in $(t, x, \varepsilon)$ in some neighbourhood of the plane $t=0$. We prove in $\S 4$ that this solution actually extends analytically in $(t, x, \varepsilon)$ to all of $\mathbb{R}^{3}$, the extension satisfying

$$
\sqrt{|f|^{2}+|g|^{2}} \leq \frac{\sqrt{5}}{2} \exp \left(\frac{5}{2} x t\right)
$$

for all $t, x$ and $\varepsilon$. 
The last inequality shows that decays in the quantum case run not faster than $\exp (\gamma t)$, where $\gamma$ does not depend on $t$. This enables us to apply the Laplace transform in the analysis of system (3.1).

Since the solution of (3.1) for $\varepsilon=0$ has an explicit analytic form, it is interesting to develop the quasi-classical approach for describing the dynamics of decays. Denote by $f_{\mathrm{cl}}, g_{\mathrm{cl}}$ the solution of (3.1) for $\varepsilon=0$. We prove in $\S 5$ that

$$
f(t, x, \varepsilon)=\sum_{k=0}^{\infty} \Psi^{k} f_{\mathrm{cl}}(t, x) \varepsilon^{k},
$$

$\Psi$ being the integro-differential operator

$$
\Psi u(t, x)=-2 \imath x \int_{0}^{t} f_{\mathrm{cl}}(t-s, x) \frac{\partial u}{\partial x}(s, x) d s .
$$

The series (3.3) converges uniformly on all subsets of $\mathbb{R}_{+} \times \mathbb{R}_{+} \times \mathbb{R}_{+}$of the form

$$
\{t \leq T\} \times\{x \leq X\} \times\left\{\varepsilon \leq\left(2 T e^{3 X T}\right)^{-1}\right\} .
$$

Hence it follows that

$$
T \sim \frac{1}{3 X} \log \frac{X}{\varepsilon}
$$

in the domain of quasi-classical approach $x / \varepsilon \gg 1$. The time of applicability of the quasi-classical approach is therefore logarithmically small, i.e., $T \sim \log 1 / \hbar$ in contrast to $T \sim 1 / \hbar^{\gamma}$ for classically stable dynamics. This is a consequence of instability of the dynamics of the classical system.

A similar result was earlier obtained in the paper [3] studying conditions of applicability of quasi-classical approximations for describing dynamics of nonlinear quantum systems whose classical limits have the property of stochastic instability.

\section{Existence of solutions}

Let us formulate the problem more precisely. By (3.1), we have the following system for approximate description of the dynamics of quantum decays

$$
\begin{aligned}
& \dot{f}=-2 \imath(x-1) f-2 \imath \varepsilon x \frac{\partial f}{\partial x}+\imath x g, \\
& \dot{g}=-\imath x f
\end{aligned}
$$

in the half-plane $(t, x) \in \mathbb{R}_{+} \times \mathbb{R}$ under the initial conditions

$$
\begin{aligned}
& f(0, x)=1 \\
& g(0, x)=0 .
\end{aligned}
$$


In fact, the domain of $x=V_{0} I / \Omega$ is $x>1 / 2$, the last condition guarantying the existence of decays by (1.9).

The principal symbol of (4.1) is given by the matrix

$$
\left(\begin{array}{cc}
\imath \tau-2 \varepsilon x \xi & 0 \\
0 & \imath \tau
\end{array}\right)
$$

with the determinant $-\tau(\tau+2 \imath \varepsilon x \xi)$. It follows that (4.1) is a mixed type system with hyperbolic degeneracy on the line $x=0$. The real characteristics of this system are lines $x=$ const, hence the Cauchy problem (4.1), (4.2) is noncharacteristic.

The system (4.1) has normal form with respect to the time variable $t$, and the coefficients of the system and the Cauchy data (4.2) are entire functions of $t, x$ and $\varepsilon$. Therefore, it fulfills the conditions of the Cauchy-Kovalevskaya Theorem, which implies that the problem (4.1), (4.2) has a real analytic solution

$$
F(t, x, \varepsilon)=\left(\begin{array}{c}
f(t, x, \varepsilon) \\
g(t, x, \varepsilon)
\end{array}\right)
$$

in some neighbourhood $U$ of the hyperplane $\{t=0\}$ in $\mathbb{R}^{3}$. The solution is unique in the class of real analytic functions. Moreover, the solution is unique in the class of continuously differentiable functions, which is due to Holmgren's uniqueness theorem.

The question arises whether the solution actually extends analytically to all of the half-space $\{t>0\}$. To treat the problem we eliminate one unknown function of the system.

Lemma 4.1 Given any entire function $\Phi_{0}(x)$, the Cauchy problem for the truncated equation

$$
\left\{\begin{aligned}
\dot{\Phi} & =-2 \imath(x-1) \Phi-2 \imath \varepsilon x \frac{\partial \Phi}{\partial x} \text { for } t>0 \\
\Phi(0) & =\Phi_{0}(x)
\end{aligned}\right.
$$

hat a unique solution which is an entire function of $(t, x, \varepsilon)$.

Proof. Since the functions we work with are entire we can change the variables by

$$
\begin{aligned}
t & =\imath z, \\
\log x & =-2 \varepsilon z+w,
\end{aligned}
$$

with $z, w \in \mathbb{R}$. For the function $u(z, w):=\Phi(\imath z, \exp (-2 \varepsilon z+w))$ the Cauchy problem (4.3) becomes

$$
\left\{\begin{aligned}
\frac{\partial u}{\partial z} & =2\left(e^{-2 \varepsilon z+w}-1\right) u \text { for } z \in \mathbb{R}, \\
u(0, w) & =\Phi_{0}\left(e^{w}\right) .
\end{aligned}\right.
$$


This latter problem has a unique entire solution which can be moreover explicitly written,

$$
u(z, w)=\Phi_{0}\left(e^{w}\right) \exp \left(\frac{1}{\varepsilon} e^{w}\left(1-e^{-2 \varepsilon z}\right)-2 z\right) .
$$

Returning to the variables $t$ and $x$ yields

$$
\Phi(t, x, \varepsilon)=\Phi_{0}(x) \exp \left(\frac{x}{\varepsilon}\left(e^{-2 \imath \varepsilon t}-1\right)+2 \imath t\right),
$$

as desired.

Note that $\Phi(t, x, \varepsilon)$ converges to $\Phi_{0}(x) \exp (-2 \imath t(x-1))$ for $\varepsilon \rightarrow 0$, as is easy to see.

From now on we tacitly assume that $\Phi_{0}=1$. By abuse of notation, we use the same letter $\Phi$ to designate the solution of (4.3) with $\Phi_{0}=1$. Set

$$
\Gamma(t, x, \varepsilon)=-\imath x \int_{0}^{t} \Phi(s, x, \varepsilon) d s .
$$

Lemma 4.2 Suppose $P$ is a continuous function of $(t, x, \varepsilon)$ in the halfspace $\mathbb{R}_{+} \times \mathbb{R} \times \mathbb{R}$. Then the solution of the Cauchy problem for the system

$$
\begin{aligned}
& \dot{f}=-2 \imath(x-1) f-2 \imath \varepsilon x \frac{\partial f}{\partial x}+P, \\
& \dot{g}=-\imath x f
\end{aligned}
$$

under initial conditions (4.2) is given by the formula

$$
\begin{aligned}
f(t, x, \varepsilon) & =\Phi(t, x, \varepsilon)+\int_{0}^{t} \Phi(t-s, x, \varepsilon) P(s, x, \varepsilon) d s, \\
g(t, x, \varepsilon) & =\Gamma(t, x, \varepsilon)+\int_{0}^{t} \Gamma(t-s, x, \varepsilon) P(s, x, \varepsilon) d s .
\end{aligned}
$$

Proof. To simplify notation, we will not indicate the dependence of $f, g$, etc. on $x$ and $\varepsilon$.

Since $\Phi(0)=1$ and $\Gamma(0)=0$ both $f$ and $g$ satisfy (4.2). Furthermore, an easy calculation shows that

$$
\begin{aligned}
\dot{f}(t) & =\dot{\Phi}(t)+\Phi(0) P(t)+\int_{0}^{t} \dot{\Phi}(t-s) P(s) d s \\
& =\dot{\Phi}(t)+\Phi(0) P(t)-\int_{0}^{t}\left(2 \imath(x-1) \Phi(t-s)+2 \imath \varepsilon x \frac{\partial}{\partial x} \Phi(t-s)\right) P(s) d s \\
& =\dot{\Phi}(t)+\Phi(0) P(t)-2 \imath(x-1)(f(t)-\Phi(t))-2 \imath \varepsilon x \frac{\partial}{\partial x}(f(t)-\Phi(t)) \\
& =-2 \imath(x-1) f(t)-2 \imath \varepsilon x \frac{\partial}{\partial x} f(t)+P(t),
\end{aligned}
$$


the last equality being a consequence of (4.3), and similarly

$$
\begin{aligned}
\dot{g}(t) & =\dot{\Gamma}(t)+\Gamma(0) P(t)+\int_{0}^{t} \dot{\Gamma}(t-s) P(s) d s \\
& =\dot{\Gamma}(t)+\Gamma(0) P(t)-\imath x \int_{0}^{t} \Phi(t-s) P(s) d s \\
& =\dot{\Gamma}(t)+\Gamma(0) P(t)-\imath x(f(t)-\Phi(t)),
\end{aligned}
$$

showing the lemma.

Lemma 4.2 allows one to reduce the Cauchy problem (4.1), (4.2) to an integral equation of Volterra type, namely

$$
\begin{aligned}
& f(t)=\Phi(t)+\imath x \int_{0}^{t} \Phi(t-s) g(s) d s, \\
& g(t)=\Gamma(t)+\imath x \int_{0}^{t} \Gamma(t-s) g(s) d s .
\end{aligned}
$$

Theorem 4.3 The problem (4.1), (4.2) has a unique solution $\{f, g\}$ which is a real analytic function of $(t, x, \varepsilon)$ on all of $\mathbb{R} \times \mathbb{R} \times \mathbb{R}$, satisfying (3.2).

Proof. Since both $\Phi$ and $\Gamma$ are entire functions of $(t, x, \varepsilon)$, the existence and uniqueness of a solution follow from the classical Volterra theory. This solution can be actually obtained by successive approximations. It remains to establish (3.2).

To this end, we apply the successive approximation method to solve the second equation of (4.7), and then we substitute $g$ to the first equation, thus obtaining $f$. For simplicity, we restrict our discussion to the case of nonnegative $t, x$ and $\varepsilon$, which involves no loss of generality. Setting $g_{0}=\Gamma$, we define iterations

$$
g_{k}(t)=\Gamma(t)+\imath x \int_{0}^{t} \Gamma(t-s) g_{k-1}(s) d s
$$

for $k=1,2, \ldots$

Since

$$
\begin{aligned}
\left|g_{0}(t)\right| & \leq x \int_{0}^{t} \exp \left(\frac{x}{\varepsilon}(\cos 2 \varepsilon s-1)\right) d s \\
& \leq x \int_{0}^{t} \exp \left(\frac{x}{\varepsilon} 2 \varepsilon s\right) d s \\
& \leq \frac{1}{2}\left(e^{2 t x}-1\right)
\end{aligned}
$$


one easily obtains by induction

$$
\begin{aligned}
\left|g_{1}(t)\right| & \leq\left(\frac{1}{2}-\frac{1}{4}\right) \varphi+\frac{1}{4} t x \psi \\
\left|g_{2}(t)\right| & \leq\left(\frac{1}{2}-\frac{1}{16}+\frac{1}{16}(t x)^{2}\right) \varphi+\frac{1}{16} t x \psi \\
\left|g_{3}(t)\right| & \leq\left(\frac{1}{2}-\frac{21}{96}\right) \varphi+\left(\frac{21}{96} t x+\frac{1}{96}(t x)^{3}\right) \psi \\
\left|g_{4}(t)\right| & \leq\left(\frac{1}{2}-\frac{63}{768}+\frac{45}{768}(t x)^{2}+\frac{1}{768}(t x)^{4}\right) \varphi+\left(\frac{63}{768} t x-\frac{2}{768}(t x)^{3}\right) \psi
\end{aligned}
$$

where

$$
\begin{aligned}
& \varphi=e^{2 t x}-1, \\
& \psi=e^{2 t x}+1 .
\end{aligned}
$$

Given any $k=1,2, \ldots$, we get

$$
\left|g_{k}(t)\right| \leq\left(c_{k, 0}+c_{k, 2}(t x)^{2}+\ldots\right) \varphi+\left(c_{k, 1} t x+c_{k, 3}(t x)^{3}+\ldots\right) \psi,
$$

where $c_{k, n}=0$ for $n>k$. The coefficients $c_{k, n}$ can actually be estimated uniformly in $k$ by

$$
\left|c_{k, n}\right| \leq \frac{1}{2} \frac{1}{2^{n}} \frac{1}{n !}
$$

for all $n$.

Letting $k \rightarrow \infty$ we deduce that the limiting function $g(t)$ fulfills the estimate

$$
\begin{aligned}
|g(t)| & \leq \frac{1}{2} \sum_{n=0}^{\infty} \frac{1}{(2 n) !}\left(\frac{t x}{2}\right)^{2 n} \varphi+\frac{1}{2} \sum_{n=0}^{\infty} \frac{1}{(2 n+1) !}\left(\frac{t x}{2}\right)^{2 n+1} \psi \\
& \leq \frac{1}{2} \cosh \frac{t x}{2}\left(e^{2 t x}-1\right)+\frac{1}{2} \sinh \frac{t x}{2}\left(e^{2 t x}+1\right)
\end{aligned}
$$

for all $t \geq 0$. Using the definitions of functions $\cosh x$ and $\sinh x$ we readily obtain

$$
\begin{aligned}
|g(t)| & \leq \frac{1}{2}\left(e^{(5 / 2) t x}-e^{-(1 / 2) t x}\right) \\
& \leq \frac{1}{2} e^{(5 / 2) t x}
\end{aligned}
$$

and

$$
\begin{aligned}
|f(t)| & \leq e^{2 t x}+x \int_{0}^{t} e^{2 x(t-s)} \frac{1}{2} e^{(5 / 2) s x} d s \\
& \leq e^{(5 / 2) t x},
\end{aligned}
$$

which implies (3.2).

As already mentioned, the solution $\{f, g\}$ of (4.1), (4.2) is also unique in the space of continuously differentiable functions. 


\section{Successive approximations}

Set

$$
A=\left(\begin{array}{cc}
-2 \imath(x-1) & \imath x \\
-\imath x & 0
\end{array}\right)
$$

and let

$$
\begin{aligned}
& \lambda_{+}=\imath(1-x)+\sqrt{2 x-1}, \\
& \lambda_{-}=\imath(1-x)-\sqrt{2 x-1}
\end{aligned}
$$

stand for the eigenvalues of the matrix $A$. The system (4.1) for $\varepsilon=0$ takes the form

$$
\dot{F}_{\mathrm{cl}}=A F_{\mathrm{cl}}
$$

with

$$
F_{\mathrm{cl}}(t, x)=\left(\begin{array}{l}
f_{\mathrm{cl}}(t, x) \\
g_{\mathrm{cl}}(t, x)
\end{array}\right)
$$

hence the solution of the Cauchy problem (4.1), (4.2) corresponding to $\varepsilon=0$ can be written in the form

$$
\begin{aligned}
F_{\mathrm{cl}} & =\sum_{k=0}^{\infty} A^{k} F_{0}(x) \frac{t^{k}}{k !}, \\
& =\left(\begin{array}{c}
e^{\lambda_{-} t}+\lambda_{+} \frac{e^{\lambda_{+} t}-e^{\lambda_{-} t}}{\lambda_{+}-\lambda_{-}} \\
-\imath \frac{e^{\lambda_{+} t}-e^{\lambda_{-}}}{\lambda_{+}-\lambda_{-}}
\end{array}\right)
\end{aligned}
$$

where

$$
F_{0}=\left(\begin{array}{l}
1 \\
0
\end{array}\right)
$$

The inequality (4.9) certainly applies to $f_{\mathrm{cl}}$, thus giving an estimate for all real $t$ and $x$. In order to derive an estimate of $F_{\mathrm{cl}}$ on all of $\mathbb{C} \times \mathbb{C}$ we need the following lemma.

Lemma 5.1 As defined above, $F_{\mathrm{cl}}$ is an entire function of $t$ and $x$, satisfying

$$
\left|F_{\mathrm{cl}}(t, x)\right| \leq \exp ((2|1-x|+|x|)|t|)
$$

for all $(t, x) \in \mathbb{C} \times \mathbb{C}$.

Proof. To shorten notation, set $z=2 \imath(1-x)$ and $w=\imath x$. An easy calculation shows that

$$
A^{k} F_{0}(x)=\left(\begin{array}{c}
c_{k} z^{k}-c_{k-2} z^{k-2} w^{2}+c_{k-4} z^{k-4} w^{4}-\ldots \\
-c_{k-1} z^{k-1} w+c_{k-3} z^{k-3} w^{3}+c_{k-5} z^{k-5} w^{5}+\ldots
\end{array}\right)
$$


where the coefficients $c_{k}, c_{k-1}, \ldots, c_{0}$ are natural numbers determined by the table

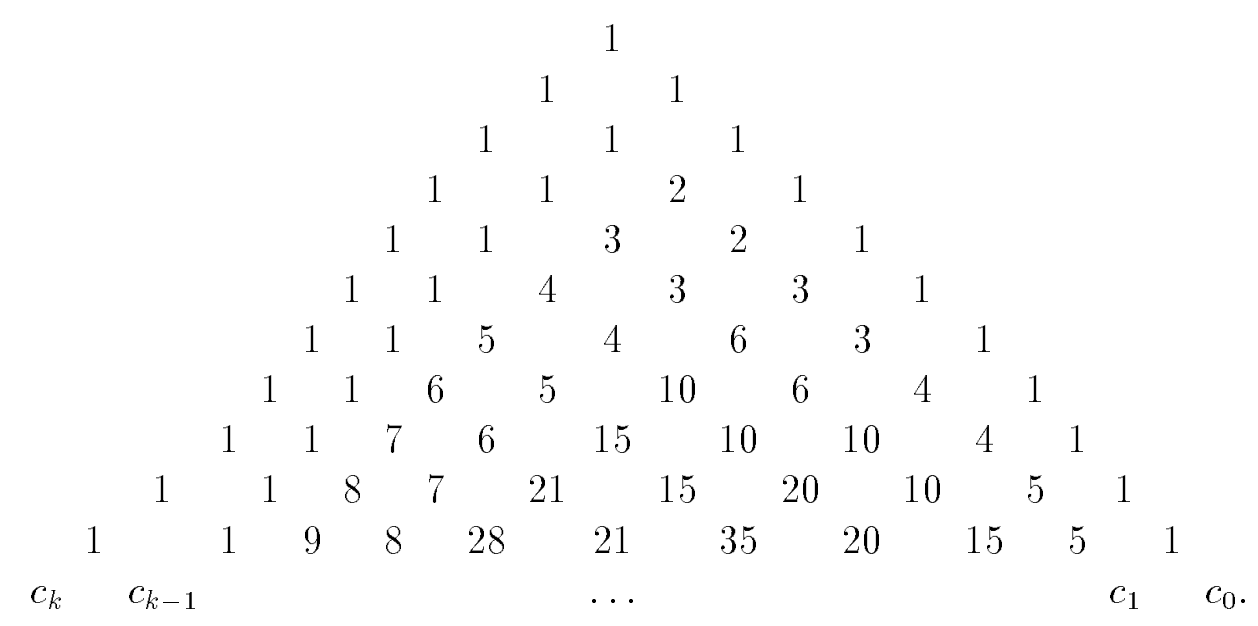

Using the inequality $\sqrt{|a|^{2}+|b|^{2}} \leq|a|+|b|$ and comparing (5.2) with the Pascal triangle we get

$$
\begin{aligned}
\left|A^{k} F_{0}(x)\right| & \leq|z|^{k}+(|z|+|w|)^{k-1}|w| \\
& \leq(|z|+|w|)^{k} .
\end{aligned}
$$

Substituting this estimate into (5.1) yields

$$
\begin{aligned}
\left|F_{\mathrm{cl}}(t, x)\right| & \leq \sum_{k=0}^{\infty}\left|A^{k} F_{0}(x)\right| \frac{|t|^{k}}{k !} \\
& \leq \exp ((|z|+|w|)|t|)
\end{aligned}
$$

for all $(t, x) \in \mathbb{C} \times \mathbb{C}$, as desired.

Theorem 4.3 shows immediately that $F(t, x, 0)=F_{\mathrm{cl}}(t, x)$ for all $t$ and $x$, i.e., the classical solution is the pointwise limit of the quantum solution if $\varepsilon \rightarrow 0$. Given any small $\varepsilon>0$, the question arises of the range of times $t$ for which the classical limit still satisfactory describes the dynamics of quantum decays.

To study the problem we make use of the geometric series to get an asymptotic expansion of $F(t, x, \varepsilon)$ in powers of $\varepsilon$.

Lemma 5.2 Let $P$ be a continuous function of $(t, x)$ in the quarter-plane $\mathbb{R}_{+} \times \mathbb{R}_{+}$. Then the solution of the Cauchy problem for the system

$$
\begin{aligned}
\dot{f} & =-2 \imath(x-1) f+\imath x g+P, \\
\dot{g} & =-\imath x f
\end{aligned}
$$


under initial conditions (4.2) is given by the formula

$$
\begin{aligned}
& f(t, x)=f_{\mathrm{cl}}(t, x)+\int_{0}^{t} f_{\mathrm{cl}}(t-s, x) P(s, x) d s, \\
& g(t, x)=g_{\mathrm{cl}}(t, x)+\int_{0}^{t} g_{\mathrm{cl}}(t-s, x) P(s, x) d s .
\end{aligned}
$$

Proof. To shorten notation, we write $f(t)$ and $g(t)$ instead of $f(t, x)$, $g(t, x)$, etc.

Since $f_{\mathrm{cl}}(0)=1$ and $g_{\mathrm{cl}}(0)=0$ both $f$ and $g$ satisfy (4.2). Furthermore, an easy calculation shows that

$$
\begin{aligned}
\dot{f}(t) & =\dot{f}_{\mathrm{cl}}(t)+f_{\mathrm{cl}}(0) P(t)+\int_{0}^{t} \dot{f}_{\mathrm{cl}}(t-s) P(s) d s \\
& =\dot{f}_{\mathrm{cl}}(t)+f_{\mathrm{cl}}(0) P(t)+\int_{0}^{t}\left(-2 \imath(x-1) f_{\mathrm{cl}}(t-s)+\imath x g_{\mathrm{cl}}(t-s)\right) P(s) d s \\
& =\dot{f}_{\mathrm{cl}}(t)+f_{\mathrm{cl}}(0) P(t)-2 \imath(x-1)\left(f(t)-f_{\mathrm{cl}}(t)\right)+\imath x\left(g(t)-g_{\mathrm{cl}}(t)\right) \\
& =-2 \imath(x-1) f(t)+\imath x g(t)+P(t),
\end{aligned}
$$

and similarly

$$
\begin{aligned}
\dot{g}(t) & =\dot{g}_{\mathrm{cl}}(t)+g_{\mathrm{cl}}(0) P(t)+\int_{0}^{t} \dot{g}_{\mathrm{cl}}(t-s) P(s) d s \\
& =\dot{g}_{\mathrm{cl}}(t)+g_{\mathrm{cl}}(0) P(t)-\imath x \int_{0}^{t} f_{\mathrm{cl}}(t-s) P(s) d s \\
& =\dot{g}_{\mathrm{cl}}(t)+g_{\mathrm{cl}}(0) P(t)-\imath x\left(f(t)-f_{\mathrm{cl}}(t)\right),
\end{aligned}
$$

which completes the proof.

Using Lemma 5.2 reduces the Cauchy problem (4.1), (4.2) to an integral equation of Volterra type, namely

$$
f(t, x, \varepsilon)=f_{\mathrm{cl}}(t, x)-2 \imath \varepsilon x \int_{0}^{t} f_{\mathrm{cl}}(t-s, x) \frac{\partial f}{\partial x}(s, x, \varepsilon) d s .
$$

As in $\S 3$, we denote by $\Psi$ the integro-differential operator

$$
\Psi u(t, x)=-2 \imath x \int_{0}^{t} f_{\mathrm{cl}}(t-s, x) \frac{\partial u}{\partial x}(s, x) d s,
$$

then the equation (5.4) can be written in the form

$$
(I-\varepsilon \Psi) f=f_{\mathrm{cl}}
$$


whence

$$
\begin{aligned}
f(t, x, \varepsilon) & =(I-\varepsilon \Psi)^{-1} f_{\mathrm{cl}}(t, x) \\
& =\sum_{k=0}^{\infty} \Psi^{k} f_{\mathrm{cl}}(t, x) \varepsilon^{k} .
\end{aligned}
$$

One verifies by induction that

$$
\Psi^{k} f_{\mathrm{cl}}(t, x)=x\left(\left(\sum_{j=0}^{k} c_{-, k, j}(x) t^{j}\right) e^{\lambda-t}+\left(\sum_{j=0}^{k} c_{+, k, j}(x) t^{j}\right) e^{\lambda_{+} t}\right)
$$

for $k=1,2, \ldots$, where $c_{ \pm, k, j}(x)$ are irrational functions having the only singularity at the point $x=1 / 2$. Since $f_{\mathrm{cl}}$ is an entire function, the iterations $\Psi^{k} f_{\mathrm{cl}}$ are entire functions of $t$ and $x$, too.

Note that (5.5) is a regular asymptotic series in powers of the small parameter. No boundary layer is required, for the degeneracy at $\varepsilon=0$ does not affect the nature of the Cauchy problem.

Theorem 5.3 The series (5.5) converges uniformly in $t, x$ and $\varepsilon$ on compact subsets of $\mathbb{R} \times \mathbb{R} \times \mathbb{R}$ of the form

$$
\{|t| \leq T\} \times\{|x| \leq X\} \times\left\{|\varepsilon| \leq\left(2 T e^{3 X T}\right)^{-1}\right\} .
$$

Proof. From the Cauchy formula it follows that if $\varphi(x)$ is an entire function of $x \in \mathbb{R}$ then

$$
\sup _{|z| \leq r^{\prime} X}\left|\frac{\partial \varphi}{\partial z}\right| \leq \frac{1}{\left(r-r^{\prime}\right) X} \sup _{|z| \leq r X}|\varphi(z)|
$$

for all $X>0$ and $0<r^{\prime}<r$.

By Lemma 5.1 we conclude that

$$
\sup _{|z| \leq r X}\left|f_{\mathrm{cl}}(t, z)\right| \leq e^{(3 r X+2)|t|}
$$

for any $r>0$. We next show by induction that for all $k=1,2, \ldots$ the estimate holds

$$
\sup _{|z| \leq X / k+1}\left|\Psi^{k} f_{\mathrm{cl}}(t, z)\right| \leq(2|t|)^{k} e^{(3 X+2)|t|} .
$$

For $k=1$ we get, by (5.6),

$$
\begin{aligned}
\sup _{|z| \leq X / 2}\left|\Psi f_{\mathrm{cl}}(t, z)\right| & \leq \sup _{|z| \leq X / 2}|-2 \imath z| \int_{0}^{t}\left|f_{\mathrm{cl}}(t-s, z)\right|\left|(\partial / \partial z) f_{\mathrm{cl}}(s, z)\right| d s \\
& \leq X \int_{0}^{t} \exp \left(\left(3 \frac{X}{2}+2\right)(t-s)\right) \frac{2}{X} \exp ((3 X+2) s) d s \\
& =2 \exp \left(\left(3 \frac{X}{2}+2\right) t\right) \int_{0}^{t} \exp \left(3 \frac{X}{2} s\right) d s \\
& \leq 2|t| \exp ((3 X+2)|t|),
\end{aligned}
$$


as desired. Having granted the inequalities (5.7) up to the number $k$, we derive, by $(5.6)$,

$$
\begin{aligned}
& \sup _{|z| \leq X / k+2}\left|\Psi^{k+1} f_{\mathrm{cl}}(t, z)\right| \\
& \leq \frac{2 X}{k+2} \int_{0}^{t} \exp \left(\left(3 \frac{X}{k+2}+2\right)(t-s)\right) \frac{(k+1)(k+2)}{X} \sup _{|z| \leq X / k+1}\left|\Psi^{k} f_{\mathrm{cl}}(s, z)\right| d s \\
& \leq 2(k+1) \exp \left(\left(3 \frac{X}{k+2}+2\right) t\right) \int_{0}^{t}(2 s)^{k} \exp \left(3 \frac{k+1}{k+2} X s\right) d s \\
& \leq 2(k+1) \exp ((3 X+2)|t|) \int_{0}^{t}(2 s)^{k} d s \\
& \leq(2|t|)^{k+1} \exp ((3 X+2)|t|),
\end{aligned}
$$

thus completing the induction step.

Since $X$ is actually arbitrary in (5.7) we easily deduce from this inequality that

$$
\sup _{|z| \leq X}\left|\Psi^{k} f_{\mathrm{cl}}(t, z)\right| \leq e^{(3 X+2)|t|}\left(2|t| e^{3 X|t|}\right)^{k}
$$

for all $t \in \mathbb{R}$. Hence it follows that the series (5.5) converges uniformly in $t, x$ and $\varepsilon$ on each compact set

$$
\{|t| \leq T\} \times\{|x| \leq X\} \times\left\{|\varepsilon| \leq\left(2 T e^{3 X T}\right)^{-1}\right\}
$$

for

$$
\begin{aligned}
|f(t, x, \varepsilon)| & \leq \exp ((3 X+2)|t|) \sum_{k=0}^{\infty}(2|\varepsilon||t| \exp (3 X|t|))^{k} \\
& \leq \frac{e^{(3 X+2)|t|}}{1-2|\varepsilon||t| e^{3 X|t|}}
\end{aligned}
$$

showing the theorem.

Theorem 5.3 implies that (5.1) is an asymptotic series in the powers of $\varepsilon$ for the solution of (4.1), (4.2) on bounded subsets of $\mathbb{R}_{t} \times \mathbb{R}_{x}$, provided that $\varepsilon$ is small enough. Let us express $T$ as function of $\varepsilon$ and $x$ from the inequality $\varepsilon \leq\left(2 T e^{3 X T}\right)^{-1}$ entering into the theorem. This will enable us to evaluate the characteristic times of applicability of the classical approximation corresponding to $\varepsilon=0$.

Corollary 5.4 Let $X / \varepsilon \gg 1$. Then $F_{\mathrm{cl}}$ approximates $F(t, x, \varepsilon)$ for small $\varepsilon$ if $t \leq T$ with

$$
T \sim \frac{1}{3 X} \log \frac{X}{\varepsilon}
$$


Proof. Rewrite the inequality $\varepsilon \leq\left(2 T e^{3 X T}\right)^{-1}$ in the form

$$
2 \varepsilon T e^{3 X T} \leq 1
$$

Since the left-hand side is an increasing function of $T \geq 0$, the set of all $T$ satisfying $(5.8)$ is an interval $\left[0, T_{0}\right]$, where $T_{0}=T_{0}(X, \varepsilon)$ is the root of the equation $2 \varepsilon T e^{3 X T}=1$.

Let us evaluate $T_{0}$. From $e^{3 X T}>1+3 X T$ it follows that $T<\left(e^{3 X T}-1\right) / 3 X$ for all $T \geq 0$. Hence $T_{1}<T_{0}<T_{2}$ where $T_{1}$ and $T_{2}$ are the unique positive solutions of the equations

$$
\begin{aligned}
2 \varepsilon \frac{e^{3 X T_{1}}-1}{3 X} e^{3 X T_{1}} & =1, \\
2 \varepsilon T_{2}\left(1+3 X T_{2}\right) & =1,
\end{aligned}
$$

respectively. The solutions of these equations can be explicitly found, more precisely,

$$
\begin{aligned}
& T_{1}=\frac{1}{3 X} \log \frac{1}{2}\left(1+\sqrt{1+6 \frac{X}{\varepsilon}}\right), \\
& T_{2}=\frac{1}{6 X}\left(-1+\sqrt{1+6 \frac{X}{\varepsilon}}\right) .
\end{aligned}
$$

The asymptotic of $T_{1}$ in the domain of quasi-classical approach $x / \varepsilon \gg 1$ is actually

$$
T_{1} \sim \frac{1}{6 X} \log \frac{X}{\varepsilon}
$$

as is easy to check.

To gain the factor 2 we invoke successive approximations for solving the equation $2 \varepsilon T e^{3 X T}=1$, or equivalently $T=F(T)$ with

$$
F(T)=\frac{1}{3 X} \log \frac{1}{2 \varepsilon T}
$$

Namely, if the initial approximation $T_{0}$ is chosen sufficiently close to the solution then the iterations $T_{k+1}=F\left(T_{k}\right)$ converge to the solution. We thus get

$$
\begin{aligned}
T_{2} & =-\frac{1}{3 X} \log \left(2 \varepsilon T_{1}\right) \\
& =-\frac{1}{3 X} \log \left(\frac{2 \varepsilon}{3 X} \log \frac{1}{2 \varepsilon T_{0}}\right) \\
& \sim \frac{1}{3 X} \log \frac{X}{\varepsilon}+o\left(\log \frac{X}{\varepsilon}\right)
\end{aligned}
$$

for $\varepsilon \rightarrow 0+$, as desired. 


\section{Asymptotic under large time}

Since the time of applicability of the quasi-classical approach is logarithmically small, the question arises on the behaviour of $f$ and $g$ for large $t$, cf. [1]. To study the problem we invoke the Laplace transform in the variable $t>0$, which is denoted by

$$
\hat{F}(\tau)=\int_{0}^{\infty} e^{-\imath \tau t} F(t) d t
$$

By Theorem 4.3, the solution $F$ of (4.1), (4.2) is of exponential growth $\exp (\gamma t)$ in $t>0$, where $\gamma=5 / 2 x$. Hence the Laplace transform of $F$ is an analytic function of $\tau$ in the lower half-plane $\Im \tau<-\gamma$. Taking into account that

$$
\hat{\dot{F}}(\tau)=-F(0)+\imath \tau \hat{F}(\tau)
$$

and applying the Laplace transform to both sides of (4.1) we reduce the problem to

$$
\begin{aligned}
2 \varepsilon x \frac{\partial \hat{f}}{\partial x}+(\tau+2(x-1)) \hat{f}-x \hat{g} & =-\imath, \\
\tau \hat{g} & =-x \hat{f} .
\end{aligned}
$$

Eliminating $\hat{g}$ from this system we arrive at the Cauchy problem for $\hat{f}$ on the half-axis $x \in \mathbb{R}_{+}$

$$
\begin{aligned}
2 \varepsilon x \frac{\partial \hat{f}}{\partial x}+\left(\frac{\tau^{2}+x^{2}}{\tau}+2(x-1)\right) \hat{f} & =-\imath, \\
\hat{f}(\tau, 0) & =\frac{-\imath}{\tau-2},
\end{aligned}
$$

the initial condition being an immediate consequence of the differential equation. Note that the term in the parentheses in the differential equation is equal to

$$
a(\tau, x):=\frac{\left(\tau+\imath \lambda_{+}\right)\left(\tau+\imath \lambda_{-}\right)}{\tau} .
$$

Note that (6.1) is a Fuchs-type equation on the half-axis. Such equations are usually treated in weighted Sobolev spaces and no boundary conditions are posed at $x=0$. In fact, we derived a condition at $x=0$ from the differential equation itself. Our approach is however justified by the fact that we deal with the solution $\hat{f}(\tau, x)$ whose existence and analyticity for all $x \in \mathbb{R}$ are known a priori.

The solution of the Cauchy problem $(6.1)$ on $\mathbb{R}_{+}$is given by a familiar formula

$$
\hat{f}(\tau, x)=\frac{-\imath}{\tau-2}\left(1-\int_{0}^{x}\left(\frac{y}{x}\right)^{\frac{\tau-2}{2 \varepsilon}} \frac{\partial}{\partial y} \exp \left(-\frac{1}{2 \varepsilon}\left(\frac{x^{2}-y^{2}}{2 \tau}+2(x-y)\right)\right) d y\right)
$$


obtained of the standard formula by a single partial integration. Further partial integration leads to an asymptotic expansion of $\hat{f}$ in powers of $\varepsilon$ which corresponds to (5.5).

In order to evaluate the inverse Laplace transform of $\hat{f}$

$$
f(t)=\frac{1}{2 \pi} \int_{\mathbb{R}-\imath \gamma} e^{\imath t \tau} \hat{f}(\tau) d \tau
$$

we expand the exponential function in (6.2) in a power series and integrate it termwise over $y \in[0, x]$. This gives

$\hat{f}=\frac{1}{\imath} \exp \left(-\frac{x}{\varepsilon}\right) \sum_{n=0}^{\infty} \frac{1}{n !}\left(\frac{x}{\varepsilon}\right)^{n} \sum_{k=0}^{n}\left(\begin{array}{l}n \\ k\end{array}\right)\left(-\frac{x}{4 \imath}\right)^{k} \sum_{j=0}^{\infty} \frac{1}{j !}\left(\frac{x^{2}}{4 \imath \varepsilon}\right)^{j} \frac{1}{\tau-c_{n, k}} \frac{1}{(\imath \tau)^{k+j}}$

where $c_{n, k}=2-2 \varepsilon(n+k)$. Hence the computation of $f$ reduces to that of integrals

$$
\mathcal{I}_{n, k, j}(t)=\frac{1}{2 \pi} \int_{\mathbb{R}-\imath \gamma} e^{\imath t \tau} \frac{1}{\imath \tau-\imath c_{n, k}} \frac{1}{(\imath \tau)^{k+j}} d \tau,
$$

which can be done by residue theory. Namely,

$$
\begin{aligned}
& \mathcal{I}_{n, 0,0}(t)=\exp \left(\imath c_{n, 0} t\right) \\
& \mathcal{I}_{n, k, j}(t)=\frac{\exp \left(\imath c_{n, k} t\right)}{\left(\imath c_{n, k}\right)^{k+j}}\left(1-\frac{\Gamma\left(k+j, \imath c_{n, k} t\right)}{(k+j-1) !}\right)
\end{aligned}
$$

where

$$
\Gamma(N, z)=(N-1) ! e^{-z} \sum_{n=0}^{N-1} \frac{z^{n}}{n !}
$$

is the incomplete gamma-function. Setting $(-1) !=\infty$ and integrating $\hat{f}$ termwise over $\tau \in \mathbb{R}-\imath \gamma$, we get

$$
f=\exp \left(-\frac{x}{\varepsilon}\right) \sum_{n=0}^{\infty} \frac{1}{n !}\left(\frac{x}{\varepsilon}\right)^{n} \sum_{k=0}^{n}\left(\begin{array}{l}
n \\
k
\end{array}\right)\left(-\frac{x}{4 \imath}\right)^{k} \sum_{j=0}^{\infty} \frac{1}{j !}\left(\frac{x^{2}}{4 \imath \varepsilon}\right)^{j} \mathcal{I}_{n, k, j}(t) .
$$

Note that the dependence on $t$ occurs only in the last sum of (6.3). We now sum up over $j=0,1, \ldots$. It is easy to check that

$$
\begin{aligned}
& \sum_{j=0}^{\infty} \frac{1}{j !}\left(\frac{x^{2}}{4 \imath \varepsilon}\right)^{j} \mathcal{I}_{n, k, j}(t) \\
= & \frac{1}{\left(\imath c_{n, k}\right)^{k}}\left(\exp \left(\imath c_{n, k} t-\frac{x^{2}}{4 c_{n, k} \varepsilon}\right)+\sum_{j=1}^{\infty} \frac{J_{j}(v)}{\left(-\imath c_{n, k} t\right)^{j}}+\sum_{j=0}^{k-1}\left(-\frac{4 c_{n, k} \varepsilon}{x^{2}}\right)^{j} J_{j}(v)\right),
\end{aligned}
$$


where $J_{j}(v)$ is Bessel's function of order $j$ and

$$
\begin{aligned}
v & =\sqrt{\imath \frac{x^{2} t}{\varepsilon}} \\
& =\sqrt{\imath\left(\frac{x}{\varepsilon}\right)^{2} \varepsilon t .}
\end{aligned}
$$

Since the quantity $v$ is complex the function $J_{j}(v)$ grows exponentially when $|v| \rightarrow \infty$.

From now on we restrict ourselves to the case $\varepsilon t \gg 1$. Then the principal term in (6.4) is

$$
\sum_{j=0}^{k-1}\left(-\frac{4 c_{n, k} \varepsilon}{x^{2}}\right)^{j} J_{j}(v)
$$

hence the expansion (6.3) takes the form

$$
f \sim \exp \left(-\frac{x}{\varepsilon}\right) \sum_{n=0}^{\infty} \frac{1}{n !}\left(\frac{x}{\varepsilon}\right)^{n} \sum_{k=0}^{n}\left(\begin{array}{l}
n \\
k
\end{array}\right)\left(\frac{x}{4 c_{n, k}}\right)^{k} \sum_{j=0}^{k-1}\left(-\frac{4 c_{n, k} \varepsilon}{x^{2}}\right)^{j} J_{j}(v) .
$$

For $|v| \gg 1$ one can invoke a familiar asymptotic of Bessel's function, more precisely

$$
J_{j}(v) \sim \sqrt{\frac{2}{v \pi}} \cos \left(v+j \pi+\frac{1}{2} \pi\right) .
$$

It follows that

$$
f(t, x, \varepsilon)=\exp \sqrt{\frac{x^{2} t}{2 \varepsilon}} Q(t, x, \varepsilon)
$$

provided that $\varepsilon t \gg 1$ and $|v| \gg 1$, where $Q$ is a slowly varying nonincreasing function of $t$.

Note that this result can be also derived by the saddle-point method. The advantage of using the above analysis of large $t$ lies in the fact that it allows one to study also other limit cases.

\section{Conclusion}

We have described the decay processes in a nonlinear quantum chain with Hamiltonian (0.2). The analysis is based on the approximate equation (1.5). This approach is justified in the framework of the so-called "narrow packet" approximation. For the classical limit the condition of "narrow packet" amounts to the fact that the parameters and initial conditions of excitation of the chain meet certain restrictions. 
The equation (1.5) is equivalent to a nonlinear Schrödinger equation with periodic boundary conditions. The latter is known to be a completely integrable system both in the classical and quantum cases, cf. [9].

In the classical limit $\hbar=0$ the four wave decays of the above type are possible merely in the domain (1.9), i.e., $x>1 / 2$. In this case the increment of instability is determined by (1.8), or

$$
\begin{aligned}
A_{k \pm l}(t) & \sim A_{k \pm l}(0) \exp \left(l \Omega \sqrt{2 x-l^{2}} t\right) \\
& \leq A_{k \pm l}(0) \exp (\Omega x t) .
\end{aligned}
$$

Quantum effects result in slowing down the development of instability for large times

$$
A_{k \pm l}(t) \sim A_{k \pm l}(0) \exp \left(\Omega x \sqrt{\frac{t}{V_{0} \hbar}}\right)
$$

provided $t \gg 1 / V_{0} \hbar$. The slowing-down of the development of instability was earlier observed in [7] where the dynamics of a quantum rotator stimulated by a periodic sequence of $\delta$-impulses was studied.

The second peculiarity of quantum effects in the dynamics of decays is the gap in the critical value of amplitude of the initial wave. In the quantum case the periodic wave (2.4) is therefore always instable.

It is also worth mentioning that the result on the applicability times of quasi-classical approach for systems (3.1) is of independent interest. Because of exponential instability of the classical system the time of applicability of the asymptotic series (5.5) in powers of $\hbar$ is logarithmically small in the quasiclassical domain $x / \varepsilon \gg 1$. 


\section{References}

[1] G. P. Berman, A. M. Iomin, A. P. Kolovskit, and N. N. Tarkhanov, On the Dynamics of the Four-Wave Interactions in a NonLinear Quantum Chain, Preprint N 377 F, Inst. of Physics, Krasnoyarsk, 1986, 45 pp.

[2] G. P. Berman, A. M. Iomin, and G. M. Zaslavskit, Method of quasi-classical approximation for $c$-number projection in coherent states basis, Physica 4 D (1981), 113-121.

[3] G. P. Berman and G. M. Zaslavskil, Quantum mappings and the problem of stochasticity in quantum systems, Physica 111 A (1982), 1744.

[4] G. P. Berman and A. P. KolovskiI, On the bound of stochasticity for a one-dimensional nonlinear chain of interacting oscillators, J. of Exper. and Theor. Physics 87 (1984), 1938-1947.

[5] G. P. Berman, A. Smerzi, and A. R. Bishop, Quantum instability of a Bose-Einstein condensate with attractive interaction, Phys. Rev. Lett. 88 (2002), no. 120402, 1-4.

[6] N. Budincky and T. BounTis, Stability of nonlinear modes and chaotic properties of $1 D$ Fermi-Pasta-Ulam lattices, Physica 8 D (1983), $445-452$.

[7] B. V. Chirikov and Th. M. Izraelev, Dynamical stochasticity in classical and quantum mechanics, Soviet Review 2 C (1981), 209-267.

[8] E. Fermi, J. Pasta and B. Ulam, Studies of Nonlinear Problems, 1955, LA-1940.

[9] A. G. Isergin and V. E. KorePin, Quantum method of inverse problem, Physics of Elementary Particles and Atom Nucleus 13 (1982), no. 3, 501-541.

[10] Yu. A. Sinitsyn and V. M. Tsukernik, Manifestation of quantum properties in evolution of physical quantities for nonlinear Hamiltonian systems, Phys. Lett. 90 A (1982), 339-341.

[11] B. E. ZaKHAROV, On the stability of waves in nonlinear environments with dispersion, J. of Exper. and Theor. Physics 51 (1966), 1107-1113.

[12] G. M. Zaslavski, Stochasticity of Dynamical Systems, Nauka, Moscow, 1984. 
Quantum Dynamics in the Fermi-Pasta-Ulam Problem

\section{E-mail addresses :}

gpb@lanl.gov

tarkhanov@math.uni-potsdam.de 